\title{
Seismic response of adjacent filled parallel rock fractures with dissimilar properties
}

\author{
W. Wu ${ }^{\text {a,* }}$, J.C. Li ${ }^{\text {b }}$, J. Zhao ${ }^{\mathrm{a}}$

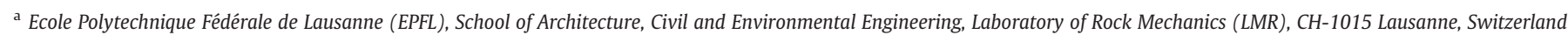 \\ b State Key Laboratory of Geomechanics and Geotechnical Engineering, Institute of Rock and Soil Mechanics, Chinese Academy of Sciences, Wuhan 430071, China
}

\section{A R T I C L E I N F O}

\section{Article history:}

Received 6 February 2013

Accepted 20 June 2013

Available online 4 July 2013

\section{Keywords:}

P-wave propagation

Filled parallel fractures

Recursive method

Specific fracture stiffness

Wave transmission coefficient

\begin{abstract}
A B S T R A C T
The purpose of this study is to analytically predict and to experimentally investigate the seismic response of adjacent filled parallel rock fractures with dissimilar properties (e.g., fracture thickness and stiffness). The time-domain recursive method is extended to predict that a P-wave propagates normally across the filled parallel fractures using the specific stiffness of each filled fracture and considering multiple wave reflections between the parallel fractures. The split Hopkinson rock bar technique is modified to simulate P-wave propagation normally across the sand-filled parallel fractures and to characterize the stress-closure relation of each sand-filled fracture. The P-wave transmission and the seismic response of the filled parallel fractures are an interactive process. The experimental results show the decreases of loading rate and dominant frequency when the P-wave propagates across each sand-filled fracture. The P-wave transmitted from the first sandfilled fracture strongly affects the seismic response of the second one. The P-wave attenuation in the filled parallel fractures is mainly due to the dynamic compaction of the filling sands. By comparison, the analytical method provides a satisfactory prediction to the experimental result. This study suggests considering the specific stiffness of each filled fracture to precisely predict the seismic response of filled parallel rock fractures.
\end{abstract}

(c) 2013 Elsevier B.V. All rights reserved.

\section{Introduction}

Seismic response of rock fractures is associated not only with a planar contact of country rock walls, but also with a gouge layer of viscoelastic materials filled between the walls. The filling gouges are found to be ubiquitous in rock fractures at all scales (Marone and Scholz, 1989). Gouge formation is mainly due to fragmentation of intact rocks exposed to sliding wear or implosive loading (Wilson et al., 2005). When an incident P-wave propagates, the seismic responses of the filled fractures largely affect how much seismic energy can travel through rock masses and how rock masses can resist the seismic disturbance. Understanding the mechanical and seismic roles of the filled fractures is thus essential to estimate seismic energy attenuation and rock mass instability (e.g., Ali and Jakobsen, 2011; Perino, 2011; Zhao et al., 1999).

A group of nearly parallel fractures in rock masses is generally known as a set. The seismic response of a set of parallel fractures has been studied using different analytical methods, such as the method of characteristics (Bedford and Drumheller, 1994; Cai and Zhao, 2000), the scattering matrix method (Aki and Richards, 2002; Perino et al., 2012) and the virtual wave source method (Li et al., 2010). Most of these methods are narrowed to non-filled parallel fractures and conclude that the seismic response of parallel fractures

\footnotetext{
* Corresponding author. Tel.: + 41 216933962; fax: + 41216934153.

E-mail address: wei.wu@epfl.ch (W. Wu).
}

depends on the ratio between incident wavelength and fracture spacing (Zhao et al., 2006). The stiffness of each fracture can be considered identically, when the fracture spacing is much smaller than the wavelength. The fracture spacing may have no effect on wave transmission, when it becomes longer than the wavelength. Between these cases, wave superposition has great effects on wave transmission. Zhu et al. (2012) modified a recursive method in the frequency domain to estimate P-wave propagation across filled parallel rock fractures. The layered medium model assumes that each filled fracture has the similar physical and mechanical properties and spatial configurations. Many previous analytical studies on the seismic response of parallel fractures are based on this assumption (e.g., Li et al., 2011; Zhao et al., 2006). However, the discrete and strongly heterogeneous gouges may induce dissimilar physical and mechanical properties of the filled fractures in a fracture set, such as fracture thickness and stiffness. It has been found that this assumption may not precisely predict the seismic response of the filled parallel fractures ( $\mathrm{Wu}$ et al., 2013a). Questions therefore remain open to the seismic response of filled parallel rock fractures with dissimilar properties.

This study analytically predicts and experimentally investigates the seismic response of adjacent filled parallel rock fractures with dissimilar properties. It studies the loading rate dependence and the dominant frequency dependence of the filled fractures in a fracture set, as well as multiple wave reflections between the parallel fractures. A P-wave is easy to be generated and measured experimentally and thus used to represent a seismic wave in this study. For 
simplification, two filled parallel fractures are considered and described as adjacent filled parallel fractures. The time-domain recursive method (TDRM) is extended to predict that a P-wave propagates normally across the filled parallel fractures with dissimilar properties. The split Hopkinson rock bar (SHRB) technique is modified to simulate P-wave propagation normally across the sand-filled parallel fractures and to characterize the stress-closure relation of each sand-filled fracture, in order to observe the changes of loading rate and dominant frequency and verify the analytical predictions.

\section{Analytical method}

The TDRM was originally proposed to effectively characterize the interaction between P-wave propagation and a set of parallel rock fractures with linearly elastic behaviors (Li et al., 2011). The TDRM can be extended for P-wave propagation across nonlinear fractures. With the known incident wave and the known mechanical properties of rock fractures, this method is practical to predict the seismic responses of non-filled and filled parallel rock fractures.

This study further develops the TDRM and overcomes the previous assumption that each fracture in a fracture set has the similar physical and mechanical properties. When an incident wave arrives at the front interface of a single fracture, the specific fracture stiffness obtained from the corresponding SHRB test is employed to calculate the related transmitted wave at the rear interface. Meanwhile, multiple wave reflections between the parallel fractures are also considered by the recursive method in the time domain.

According to the one-dimensional wave propagation theory, two waves propagate along and opposite to the loading direction, which are denoted as a positive wave and a negative wave, respectively. When an incident P-wave normally impinges adjacent parallel fractures along the loading direction, the P-wave propagation equations across the 1 st or 2 nd fracture (see Fig. 1 inset) can be simplified from the deviation by Li et al. (2011) and expressed as the differential form,

$v^{n-}\left(t_{i}, J\right)=-v^{p-}\left(t_{i}, J\right)+v^{n+}\left(t_{i}, J\right)+v^{p+}\left(t_{i}, J\right)$

$\begin{aligned} v^{p+}\left(t_{i+1}, J\right)= & -v^{n+}\left(t_{i+1}, J\right)+\left(1-k_{n} \Delta t / z\right) v^{p+}\left(t_{i}, J\right)+\left(1+k_{n} \Delta t / z\right) v^{n+}\left(t_{i}, J\right) \\ & +k_{n} \Delta t / z v^{p-}\left(t_{i}, J\right)-k_{n} \Delta t / z v^{n-}\left(t_{i}, J\right)\end{aligned}$

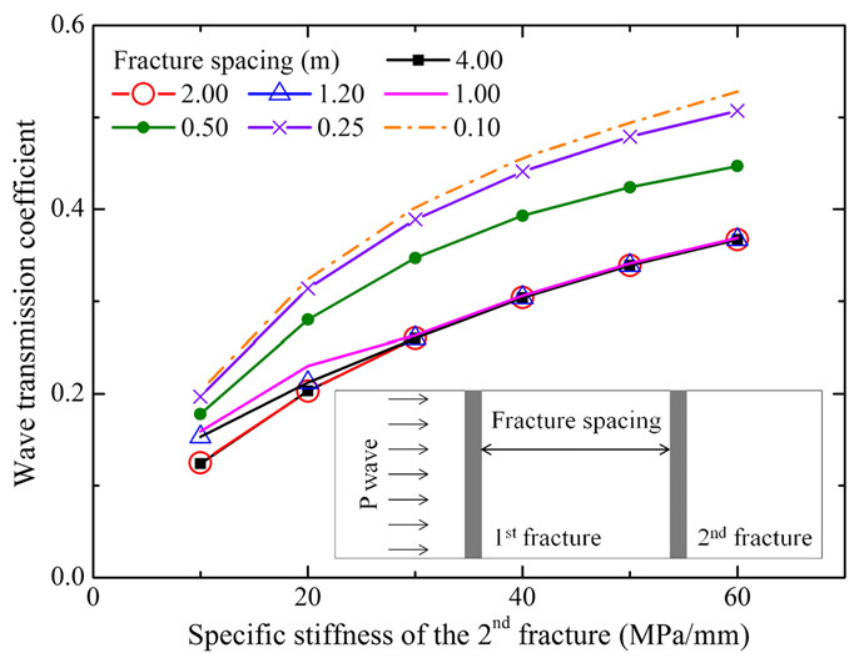

Fig. 1. Analytical prediction on the seismic response of adjacent parallel filled rock fractures. The specific stiffness of the 1st fracture keeps constant (60 MPa/mm). where $J=1,2$ for the 1 st, 2 nd fracture, respectively, $v^{p-}(t)$ and $v^{p+}(t)$ are the particle velocities at the front and rear interfaces along the loading direction, respectively, $v^{n-}(t)$ and $v^{n+}(t)$ are the particle velocities at the front and rear interfaces opposite to the loading direction, respectively, $\Delta t$ is a small time interval, $k_{n}$ is the specific fracture stiffness, and $z$ is the P-wave impedance and equal to the rock density, $\rho$, multiplied by the P-wave velocity in the rock medium, $c$. The rock medium between the parallel fractures is known as fracture spacing, $S$. The particle velocities across the fracture spacing can be written as the time-shifting functions,

$v^{p-}\left(t_{i}, J\right)=v^{p+}\left(t_{i}-S / c, J-1\right)$

$v^{n+}\left(t_{i}, J\right)=v^{n-}\left(t_{i}-S / c, J+1\right)$.

Eqs. (1) and (2) show the P-wave propagation across a single fracture and Eqs. (3) and (4) are applied for the P-wave propagation between the parallel fractures. From Eqs. (1) to (4), multiple wave reflections between the parallel fractures are considered. With the initial conditions, $v^{p-}\left(t_{1}, J\right), v^{p+}\left(t_{1}, J\right), v^{n-}\left(t_{1}, J\right)$ and $v^{n+}\left(t_{1}, J\right)$ for each fracture, and the boundary conditions, $v^{p-}\left(t_{i}, 1\right)$ for the 1 st fracture, Eqs. (1) to (4) are applied to determine the reflected wave $v^{n-}$ $\left(t_{i}, 1\right)$ for the 1 st fracture and the transmitted wave $v^{p+}\left(t_{i}, 2\right)$ for the 2nd fracture, where $v^{p-}\left(t_{i}, 1\right)$ is assumed as an incident P-wave. The incident wave from the experimental data is expressed as the strain-time response, $\varepsilon(t)$, and needs to be converted to the particle velocity-time response, $v(t)$, in the analytical calculation, where $\varepsilon(t)=v(t) / c$. The method is established in the time domain. There is no need to involve other mathematical methods, such as the Fourier and the inverse Fourier transforms. The calculating efficiency is thus improved.

In the calculation process, the positive wave at the front interface of the 1st fracture from the corresponding test is used as the incident P-wave for calculation. When a positive wave arrives at the front interface of a filled fracture, the specific fracture stiffness from the corresponding test is applied to calculate the positive wave at the rear interface. The positive wave at the rear interface of the 2nd fracture is then obtained as the transmitted wave after the filled parallel fractures. The wave transmission coefficient is defined as the ratio of the maximum strain of the positive wave at the rear interface of the 2nd fracture to that of the corresponding positive wave at the front interface of the 1 st fracture in the time domain.

Fig. 1 shows the analytical prediction on the seismic response of adjacent filled parallel fractures. The specific stiffness of the 1st fracture keeps constant, $60 \mathrm{MPa} / \mathrm{mm}$. The incident wave measured from the test N06, which is the positive wave at the front interface of the 1st fracture (shown as the incident wave in Fig. 4a), is used for this calculation. The test N06 is an SHRB test performed on the filled parallel fractures with the 1st fracture of $2 \mathrm{~mm}$ thickness and the 2nd fracture of $4 \mathrm{~mm}$ thickness, as shown in Table 1 . The wave transmission coefficient generally increases with increasing specific stiffness of the 2nd fracture and with smaller fracture spacing between the filled parallel fractures. In this case, when the fracture spacing becomes longer than $1 \mathrm{~m}$ and the specific stiffness of the 2nd fracture is larger than $30 \mathrm{MPa} / \mathrm{mm}$, the fracture spacing has no obvious effect on the wave transmission coefficient. Hence, the wave transmission coefficient of each filled fracture in the fracture set can be considered individually. When the fracture spacing is smaller than $0.1 \mathrm{~m}$, the fracture set may be treated as a single fracture compared with the incident wavelength of $6 \mathrm{~m}$. From this figure, it is observed that if the specific stiffness of the 2nd fracture becomes smaller than that of the 1st fracture, the assumption that each filled fracture has the similar physical and mechanical properties may cause an overestimation of the wave transmission coefficient. 
Table 1

Summary of analytical predictions and experimental results.

\begin{tabular}{|c|c|c|c|c|c|c|c|}
\hline \multirow[t]{2}{*}{$\begin{array}{l}\text { Fracture } \\
\text { configuration }\end{array}$} & \multirow[t]{2}{*}{ Test no. } & \multicolumn{2}{|c|}{ Thickness (mm) } & \multicolumn{2}{|c|}{$\begin{array}{l}\text { Specific stiffness } \\
(\mathrm{MPa} / \mathrm{mm})\end{array}$} & \multicolumn{2}{|c|}{$\begin{array}{l}\text { Wave } \\
\text { transmission } \\
\text { coefficient }\end{array}$} \\
\hline & & $\begin{array}{l}1 \text { st } \\
\text { fracture }\end{array}$ & $\begin{array}{l}\text { 2nd } \\
\text { fracture }\end{array}$ & $\begin{array}{l}1 \text { st } \\
\text { fracture }\end{array}$ & $\begin{array}{l}\text { 2nd } \\
\text { fracture }\end{array}$ & SHRB & TDRM \\
\hline \multirow[t]{5}{*}{ Group 1} & NOO & 2 & 2 & 60.072 & 19.933 & 0.192 & 0.211 \\
\hline & N01 & 2 & 2 & 60.247 & 19.925 & 0.197 & 0.212 \\
\hline & N04 & 2 & 2 & 59.446 & 18.745 & 0.182 & 0.223 \\
\hline & N10 & 2 & 2 & 59.380 & 19.517 & 0.200 & 0.232 \\
\hline & N14 & 2 & 2 & 59.899 & 21.544 & 0.204 & 0.206 \\
\hline \multirow[t]{5}{*}{ Group 2} & N02 & 2 & 4 & 60.847 & 5.989 & 0.119 & 0.100 \\
\hline & N05 & 2 & 4 & 61.022 & 5.472 & 0.113 & 0.102 \\
\hline & N06 & 2 & 4 & 61.846 & 5.846 & 0.115 & 0.108 \\
\hline & N08 & 2 & 4 & 61.876 & 6.367 & 0.122 & 0.116 \\
\hline & N12 & 2 & 4 & 60.222 & 6.468 & 0.106 & 0.102 \\
\hline \multirow[t]{5}{*}{ Group 3} & N03 & 4 & 2 & 16.087 & 14.803 & 0.131 & 0.139 \\
\hline & N07 & 4 & 2 & 16.658 & 14.904 & 0.136 & 0.152 \\
\hline & N09 & 4 & 2 & 16.405 & 13.115 & 0.122 & 0.148 \\
\hline & N11 & 4 & 2 & 16.939 & 13.856 & 0.141 & 0.142 \\
\hline & N13 & 4 & 2 & 17.584 & 14.723 & 0.142 & 0.138 \\
\hline
\end{tabular}

\section{Experimental study}

Fig. 2 shows the experimental configuration of P-wave propagation across the sand-filled parallel rock fractures. The SHRB configuration consists of a loading system with a norite striker bar, a norite threebar system with two gouge layers and a LabVIEW data acquisition system. This configuration is similar to the one used in the previous study (Wu et al., 2013a). However, the previous study treated the filled parallel fractures as an entirety, in which the waveforms before the 1st fracture and after the last one can be observed. This study further develops the application of the SHRB technique by introducing a center bar between the incident bar and the transmitted bar, in order to obtain the P-wave before and after each filled fracture and to characterize the stress-closure relation of each filled fracture. The length of the center bar is $1 \mathrm{~m}$ and two strain gauge groups (SGGs) connected in a Wheatstone full bridge are mounted on the this bar. The measured data from the center bar, as well as that from the incident and transmitted bars, is separated into the positive and negative waves (Zhao and Gary, 1997). The positive and negative waves at the fracture interfaces can be derived by time-shifting the positive and negative waves from the strain gauge stations, respectively. The transmitted stress-time response, $\sigma^{-}(t)$, and the fracture closure-time response, $\Delta u(t)$, are finally obtained according to the positive and negative waves at the fracture interfaces,

$\sigma^{-}(t)=E\left(\varepsilon^{p-}(t)+\varepsilon^{n-}(t)\right)$

$\Delta u(t)=c \int_{0}^{t}\left[\left(\varepsilon^{p-}(t)-\varepsilon^{n-}(t)\right)-\left(\varepsilon^{p+}(t)-\varepsilon^{n+}(t)\right)\right] d t$

where $E$ is the Young's modulus of the norite material, $63.6 \mathrm{GPa}, c$ is the longitudinal wave velocity in the norite medium, $6000 \mathrm{~m} / \mathrm{s}, \varepsilon^{p-}(t)$ and $\varepsilon^{p+}(t)$ are the positive waves at the front and rear interfaces, respectively, and $\varepsilon^{n-}(t)$ and $\varepsilon^{n+}(t)$ are the negative waves at the front and rear interfaces, respectively.
More details regarding the apparatus and the data analysis method used can be found in Wu et al. (2012).

The dry quartz sands were used to simulate the filling gouge, because of zero viscosity and a single mineral composition. The quartz sands in the size range of $1-2 \mathrm{~mm}$ and with a bulk density of $1572 \mathrm{~kg} / \mathrm{m}^{3}$ were initially filled into pre-set gaps (2 mm or $4 \mathrm{~mm}$ ) between the bars. Two aluminum confining boxes held two sand layers at two sides of the center bar during the test and made the filling sands in a uniaxial strain state.

Because the fracture spacing is $1 \mathrm{~m}$ long, it is necessary to estimate the P-wave attenuation in the norite material before the SHRB test on the filled parallel fractures. A norite bar was prepared and four groups of strain gauges were installed on this bar (Fig. 3 inset). A P-wave was generated from the impact between the striker bar and the front end of the norite bar. The data recorded by the SGG a and the SGG b was used to calculate the positive wave at the station of the SGG a, while that recorded by the SGG c and the SGG d was used to calculate the positive wave at the station of the SGG c. Fig. 3 shows that the first peak of the positive wave from the SGG c is $1.69 \%$ smaller than that of the positive wave from the SGG a. The P-wave peak decrease is much smaller than that after a filled fracture (Fig. 4a). This figure also shows that the dominant frequency has no change when the P-wave propagates from the SGG a to the SGG c. The distance between the SGG a and the SGG $\mathrm{c}$ is about $1 \mathrm{~m}$. Therefore, the P-wave attenuation in the norite material can be neglected and this study can focus on the P-wave attenuation due to the sand-filled parallel fractures.

\section{Results and discussion}

Table 1 lists the analytical predictions and the experimental results of each test. There are three fracture configurations of the sand-filled parallel fractures, namely, group 1: $2 \mathrm{~mm}$ (the 1st fracture) and $2 \mathrm{~mm}$ (the 2nd fracture), group 2: $2 \mathrm{~mm}$ (the $1 \mathrm{st}$ fracture) and $4 \mathrm{~mm}$ (the 2nd fracture) and group 3: $4 \mathrm{~mm}$ (the 1st fracture) and $2 \mathrm{~mm}$ (the 2nd fracture). The fracture specific stiffness is estimated as the slope of the pre-peak linear portion of the stress-closure relation of a single filled fracture. The wave transmission coefficient for the filled parallel fractures is predicted by the TDRM calculation and measured from the SHRB test. By comparison, the analytical prediction provides a highly satisfactory correlation with the experimental result. Therefore, the extended TDRM improves the analytical prediction on the P-wave propagation across adjacent filled parallel fractures with the consideration of dissimilar properties of the filled parallel fractures.

Fig. 4a exhibits the loading rate change of the transmitted waves after each sand-filled fracture in a fracture set. Test N06 is taken as an example. The loading rate is determined by the gradient of the pre-peak linear portion of the positive wave at the fracture interface. In the pre-peak portion of the first loading, there is a pure positive wave at each fracture interface. After the peak point, the positive wave is superposed with late-arriving waves reflected from fracture interfaces, which is not taken into account. It is observed that the loading rate decreases from $1.5461 / \mathrm{s}$ to $1.0821 / \mathrm{s}$ when the P-wave propagates across the 1 st fracture and to $0.1631 / \mathrm{s}$ when it propagates across the 2nd fracture. The reduction of the loading rate is mainly due to the dynamic compaction of the filling sands. Firstly, the sand compaction extends the loading duration. The dynamic load induces

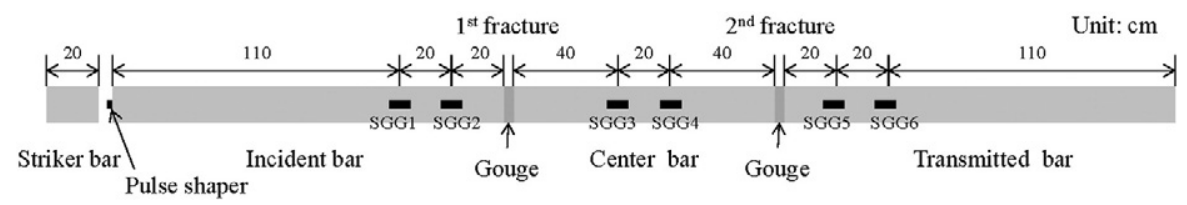

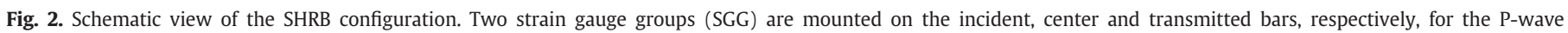
measurement. 


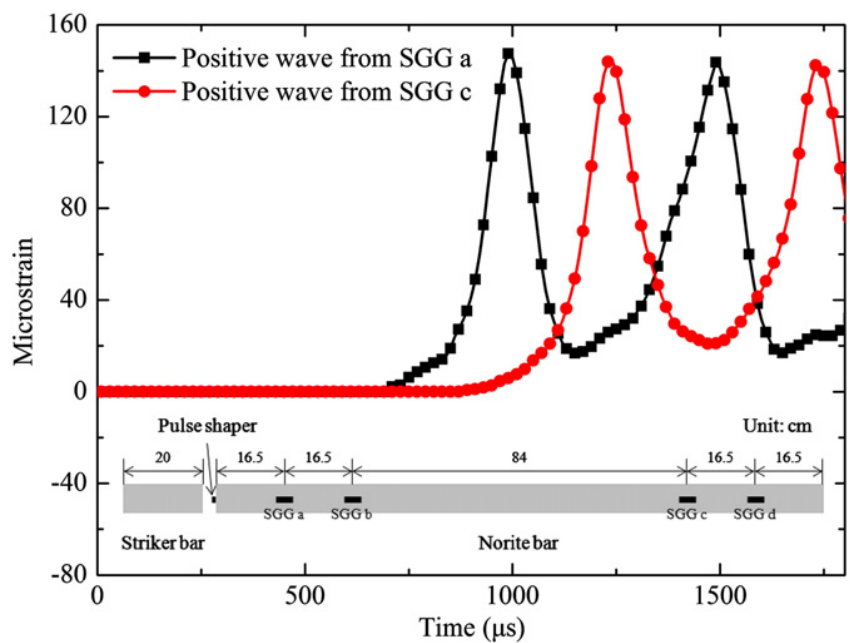

Fig. 3. P-wave attenuation in the norite material. The inset shows the experimental configuration of the P-wave measurement.

sand contacts from the elastic deformation of particle asperities to an inhomogeneous network in the form of stress chains (Majmudar and Behringer, 2005). The process continues with increasing load and takes a longer loading time. Secondly, the incident energy is remarkably consumed during the sand compaction. The maximum strain of the P-wave continuously decreases when the P-wave passes through each filled fracture.
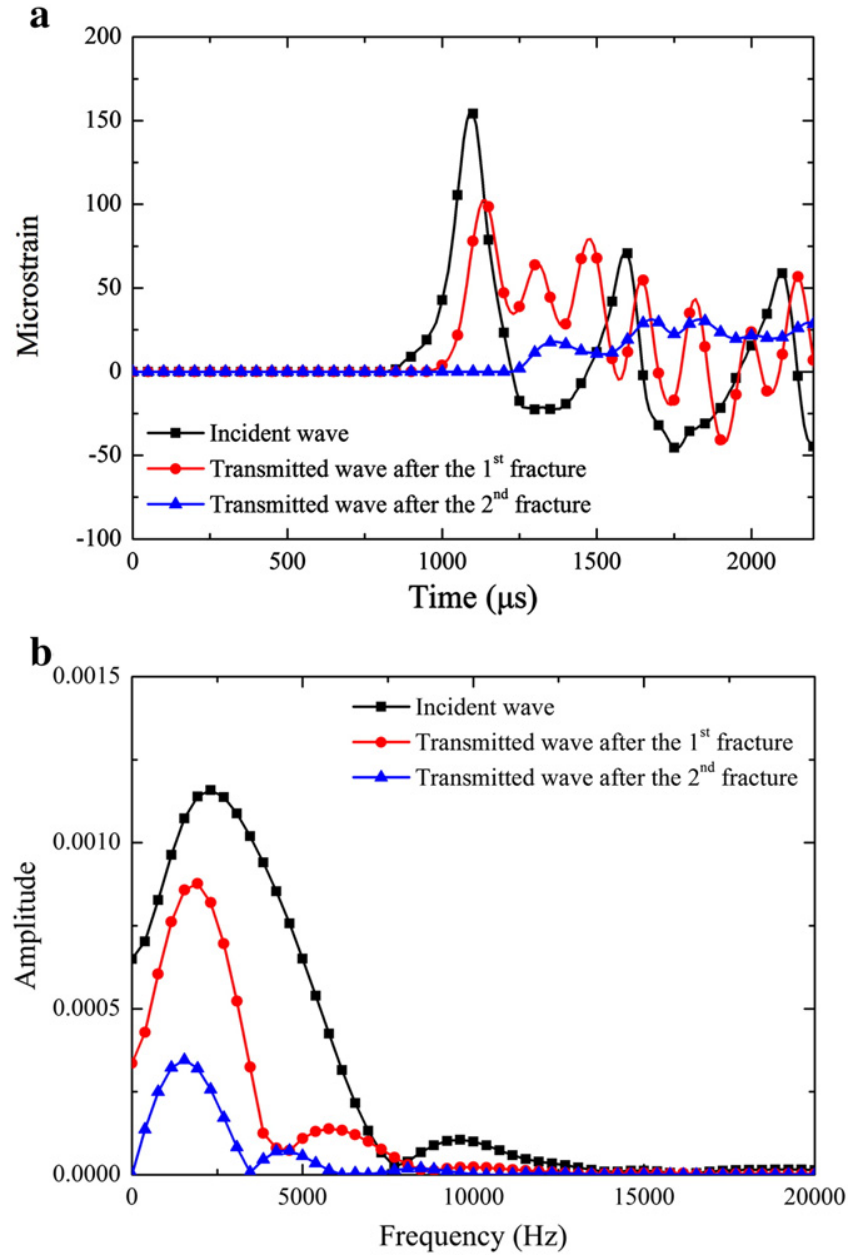

Fig. 4. P-wave propagation normally across adjacent parallel filled fractures with dissimilar properties. (a) The change of loading rate. (b) The variation of dominant frequency.
The dominant frequency change of the transmitted waves after each sand-filled fracture in the fracture set is shown in Fig. 4b. Test N06 is also taken as the example. The fast Fourier transform is used to transform the measured P-wave expressed in the time domain to that expressed in the frequency domain. The high frequency components are filtered out by the sand-filled fractures. The fracture acting as a low-pass filter has been addressed in previous studies (Pyrak-Nolte et al., 1990; Zhao et al., 2006). For the low frequency components, the amplitude at the dominant frequency decreases during the sand compaction. The dominant frequency of the P-wave at the front interface of the 1st fracture is $2308 \mathrm{~Hz}$. The dominant frequency turns to $1923 \mathrm{~Hz}$ at the rear interface of the 1 st fracture and to $1538 \mathrm{~Hz}$ at the rear interface of the 2 nd fracture. The reason for the dominant frequency change is also due to the extension of the loading duration. The sand compaction delays the P-wave arrival time at the rear interface of each filled fracture and consumes a considerable amount of the incident energy.

The changes of loading rate and dominant frequency when a P-wave propagates across rock fractures have been observed in the previous studies, for instance, Pyrak-Nolte et al. (1990) for nonfilled parallel rock fractures and $\mathrm{Wu}$ et al. (2013a) for filled parallel rock fractures. The present observation is to show that how the P-wave transmitted from the 1 st fracture affects the seismic response of the 2nd one and to verify that the specific stiffness of each filled fracture in a fracture set needs to be considered for a precise prediction on P-wave transmission.

Fig. 5 presents three typical stress-closure relations of the filled fractures in these fracture configurations. For the group 1 (e.g., the test N04), when the sand-filled parallel fractures have the same thickness, the specific stiffness of the 2nd fracture is remarkably reduced compared with that of the 1st fracture. For the group 2 (e.g., the test N06), when the thickness of the 2nd fracture becomes larger than that of the 2 nd fracture in the group 1 (the thickness of the $1 \mathrm{st}$ fracture keeps constant in two groups), the specific stiffness of the 2nd fracture is further decreased. For the group 3 (e.g., the test N07), when the thickness of the 1st fracture becomes larger than the 2nd one, the specific stiffness of the 2nd fracture is close to that of the 1 st one. The experimental results show that the specific stiffness of each sand-filled fracture depends not only on the fracture properties (e.g., thickness), but on the loading conditions (e.g., loading rate and dominant frequency).

The analytical predictions and the experimental results of the wave transmission coefficient are summarized in Fig. 6. It indicates that the wave transmission coefficient is strongly related to the specific stiffness of each filled fracture in the fracture set. A linear relation

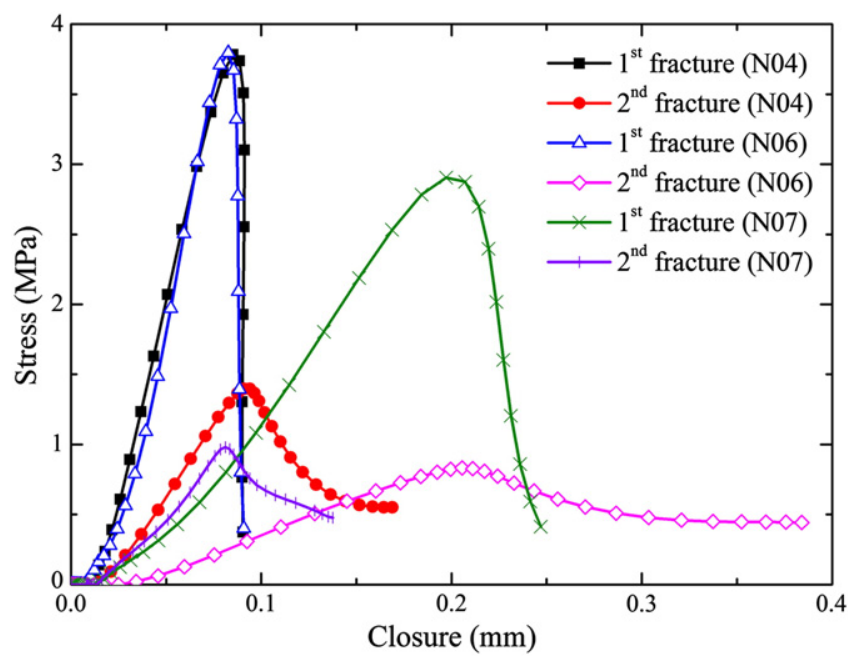

Fig. 5. Typical stress-closure relations of each filled fracture in a fracture set. 


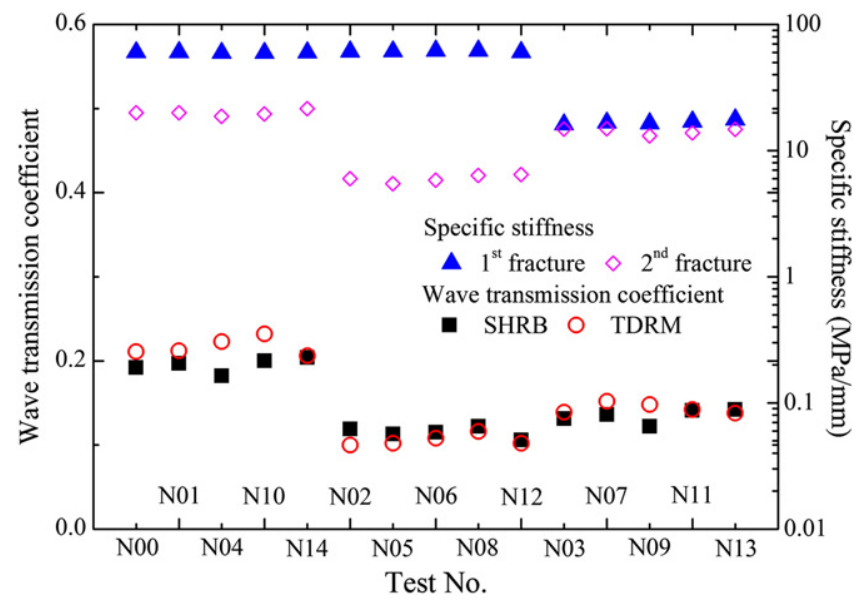

Fig. 6. Analytical predictions and experimental results of wave transmission coefficient as a function of fracture stiffness.

has been found between the wave transmission coefficient and the fracture specific stiffness of a single filled fracture, regardless of the fracture thickness, the particle size or the loading rate (Wu et al., 2013b). The fracture specific stiffness is thus a key parameter in interrelating the physical, mechanical and seismic properties of a single filled fracture. The specific stiffness of each filled fracture affects the wave transmission coefficient for the filled parallel fractures together. It can also be observed that the wave transmission coefficient for the filled parallel fractures increases with increasing specific stiffness of each filled fracture.

The experimental results extend the understanding of the seismic response of the filled parallel fractures. For the filled fractures with the same thickness, the loading rate and the dominant frequency change after the P-wave propagation across the 1st fracture, resulting in the low specific stiffness of the 2 nd one. For the filled fractures with the similar specific stiffness, the filled fractures may not have the same thickness. This observation shows that the seismic response of the parallel filled fractures in a fracture set is rate-dependent and frequency-dependent. In the previous studies, it assumes that the seismic response of each filled fracture has the same specific stiffness, if it has the same thickness and contains the same gouge material (Wu et al., 2013a). The assumption may be acceptable when the fracture spacing is much smaller than the incident wavelength (Zhao et al., 2006) and when the specific stiffness of each filled fracture is difficult to be determined. However, Fig. 1 reveals that this assumption may cause an overestimation of the wave transmission coefficient. The extended TDRM is able to consider the specific stiffness of each filled fracture in a fracture set, as well as multiple wave reflections between the parallel fractures. It calculates in the time domain, in which the data is measured from the experimental study. Therefore, the extended TDRM exhibits satisfactory predictions to the experimental results.

This study considers the changes of loading rate and dominant frequency during the first loading on the filled parallel fractures and neglects the changes of loading rate and dominant frequency during multiple wave reflections. The experimental results show that the loading rate and the dominant frequency are mainly reduced during the first loading. The multiple wave reflections between the parallel fractures make the positive wave too complicated to be separated.

\section{Conclusions}

The study provides the analytically prediction and the experimentally investigation on the seismic response of adjacent filled parallel rock fractures with dissimilar properties. The analytical method considers the specific stiffness of each filled fracture in a fracture set, as well as multiple wave reflections between the parallel fractures. The experimental observation of the P-wave propagation normally across the sand-filled parallel fractures reveals the reductions of loading rate and dominant frequency when the P-wave propagates across each sand-filled fracture. The P-wave transmitted from the 1st sand-filled fracture strongly affects the seismic response of the 2 nd one. The dynamic compaction of the gouge material in the filled fractures is responsible for the rate and frequency changes. In comparison with the analytical predictions and the experimental results, the extended TDRM can effectively predict that a P-wave propagates normally across the filled parallel fractures.

When multiple wave reflections between the filled parallel fractures greatly influence wave transmission, it is necessary to consider the specific stiffness of each filled fracture in a fracture set for a precise prediction on wave transmission. For a fracture set with a random number of filled fractures, it could be analytically and numerically investigated based on the analytical model and the experimental evidence presented in this study. The experimental study provides a basic understanding of the seismic response of the filled parallel fractures from the simplest case, but it becomes complicated if three or more filled fractures considered in a fracture set due to wave reflections from each fracture interface. The findings here are useful in evaluating the seismic response of rock masses and interpreting the logging data from site investigation.

\section{Acknowledgment}

This work is sponsored by the Swiss National Science Foundation (200021_124846).

\section{References}

Aki, K., Richards, P.G., 2002. Quantitative Seismology, 2nd ed. University Science Books. Ali, A., Jakobsen, M., 2011. Seismic characterization of reservoirs with multiple fracture sets using velocity and attenuation anisotropy data. Journal of Applied Geophysics $75,590-602$

Bedford, A., Drumheller, D.S., 1994. Introduction to Elastic Wave Propagation. John Wiley \& Sons.

Cai, J.G., Zhao, J., 2000. Effects of multiple parallel fractures on apparent attenuation of stress waves in rock masses. International Journal of Rock Mechanical and Mining Sciences 37, 661-682.

Li, J.C., Ma, G.W., Zhao, J., 2010. An equivalent viscoelastic model for rock mass with parallel joints. Journal of Geophysical Research 115, B03305.

Li, J.C., Li, H.B., Ma, G.W., Zhao, J., 2011. A time-domain recursive method to analyse transient wave propagation across rock joints. Geophysical Journal International 188, 631-644.

Majmudar, T.S., Behringer, R.P., 2005. Contact force measurements and stress-induced anisotropy in granular materials. Nature 435, 1079-1082.

Marone, C. Scholz, C.H., 1989. Particle-size distribution and microstructures within simulated fracture gouge. Journal of Structural Geology 11, 799-814.

Perino, A., 2011. Wave Propagation Through Discontinuous Media in Rock Engineering. PhD Thesis Politecnico di Torino, Italy.

Perino, A., Orta, R., Barla, G., 2012. Wave propagation in discontinuous media by the scattering matrix method. Rock Mechanics and Rock Engineering 45, 901-918.

Pyrak-Nolte, L.J., Myer, L.R., Cook, N.G.W., 1990. Transmission of seismic waves across single natural fractures. Journal of Geophysical Research 95, 8617-8638.

Wilson, B., Dewers, T., Reches, Z., Brune, J., 2005. Particle size and energetic of gouge from earthquake rupture zones. Science 434, 749-752.

Wu, W., Li, J.C., Zhao, J., 2012. Loading rate dependency of dynamic responses of rock joints at low loading rate. Rock Mechanics and Rock Engineering 45, 421-426.

Wu, W., Zhu, J.B., Zhao, J., 2013a. A further study on seismic response of a set of parallel rock fractures filled with viscoelastic materials. Geophysical Journal International 192, 671-675.

Wu, W., Zhu, J.B., Zhao, J., 2013b. Dynamic response of a rock fracture filled with viscoelastic materials. Engineering Geology 160, 1-7.

Zhao, H. Gary, G., 1997. A new method for the separation of waves: application to the SHPB technique for an unlimited duration of measurement. Journal of the Mechanics and Physics of Solids 45, 1185-1202.

Zhao, J., Zhou, Y.X., Hefny, A.M., Cai, J.G., Chen, S.G., Li, H.B., Liu, J.F., Jain, M., Foo, S.T., Seah, C.C., 1999. Rock dynamics research related to cavern development for ammunition storage. Tunnelling and Underground Space Technology 14, 513-526.

Zhao, J., Zhao, X.B., Cai, J.G., 2006. A further study of P-wave attenuation across parallel fractures with linear deformational behavior. International Journal of Rock Mechanics and Mining Sciences 43, 776-788.

Zhu, J.B., Zhao, X.B., Wu, W., Zhao, J., 2012. Wave propagation across rock joints filled with viscoelastic medium using modified recursive method. Journal of Applied Geophysics 86, 82-87. 